\title{
Stem cell therapy for myocardial infarction
}

\begin{abstract}
Clinical trials of cell therapy for the infarcted myocardium

Advances in stem cell biology have challenged the notion that infarcted myocardium is irreparable. The pluripotent ability of stem cells to differentiate into specialized cell lines began to garner intense interest within cardiology when it was shown in animal models that intramyocardial injection of bone marrow stem cells, or the mobilization of bone marrow stem cells with spontaneous homing to myocardium, could improve cardiac

$\infty$ function and survival after in$\exists$ duced myocardial infarction. ${ }^{1,2}$

Furthermore, the existence of

stem cells in myocardium has

$\widehat{c}$ been identified in animal heart,

ㄴ. and intense research is under way

$\because$ in an attempt to clarify their po-

tential clinical application for pa-
\end{abstract}

tients with myocardial infarction.

A fundamental problem in this area of research has been to determine the best source of stem cells. To date, only autologous stem cells have been studied; these have been derived from skeletal myoblasts, bone marrow and peripheral blood. Skeletal myoblasts isolated from muscle biopsy and cultured ex vivo are under clinical investigation, but they cannot transdifferentiate into authentic cardiomyocytes and have been suggested to be potentially arrhythmogenic. ${ }^{3}$ In several early clinical trials, investigators used bone marrow and peripheral blood stem cells. These were heterogeneous and relatively unselected and probably contained hematopoietic stem cells, mesenchymal stem cells and en-

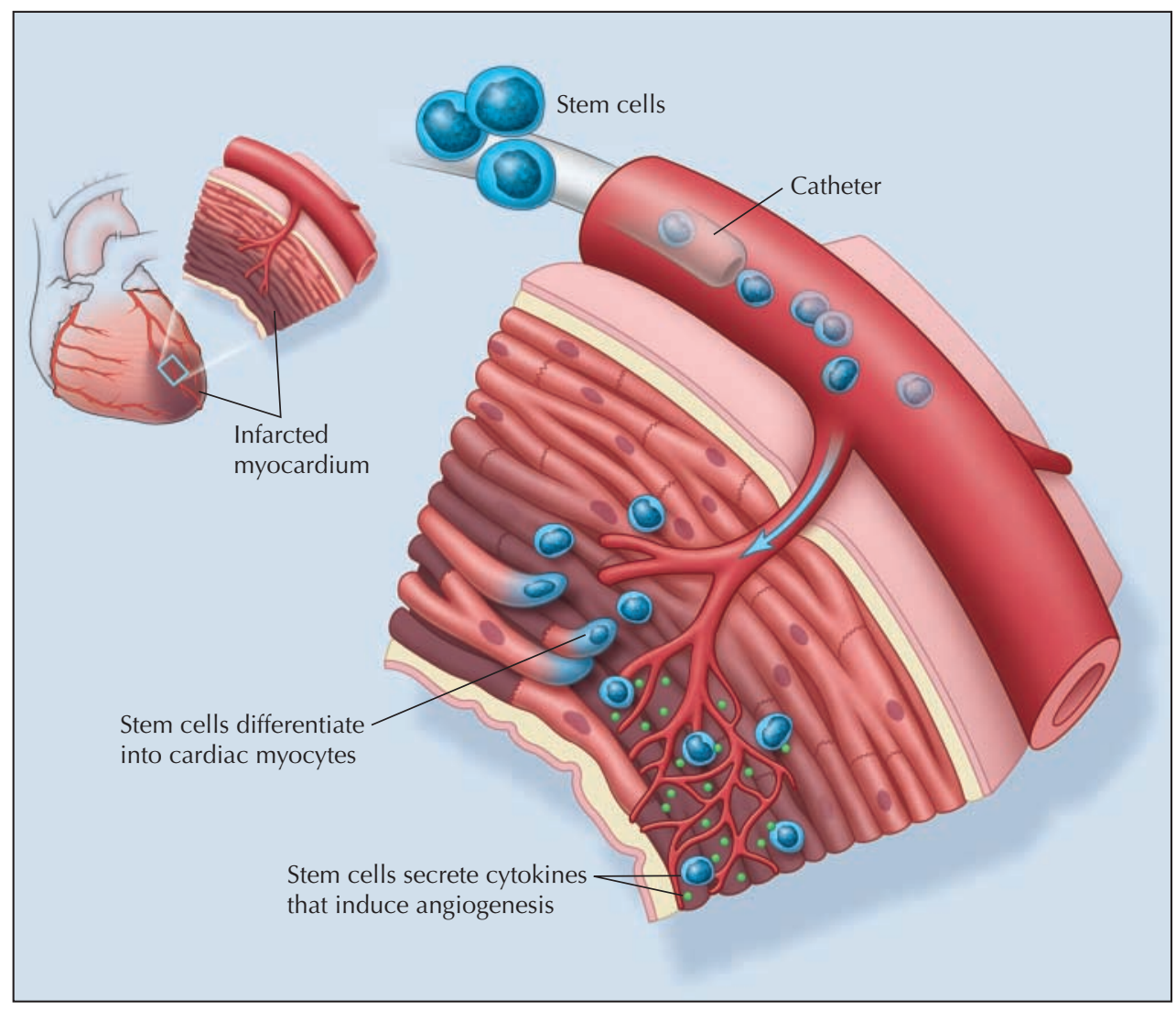

Stem cells are delivered to the heart through an intracoronary catheter. Once the stem cells have reached areas of infarcted myocardium, they may improve contractility by differentiating into functional cardiac myocytes and improve perfusion by releasing cytokines that induce angiogenesis. dothelial progenitor cells. Although bone marrow may be the richest source for stem cells with the potential to differentiate into cardiomyocytes and blood vessels, the invasiveness of bone marrow harvesting is problematic. The mobilization of peripheral blood stem cells from bone marrow could be a practical alternative that would avoid invasive bone marrow aspiration and the arrhythmogenicity associated with skeletal myoblasts, but this also has adverse effects. Granulocyte colony stimulating factor (G-CSF) is frequently used to mobilize marrow stem cells but is also associated with mobilization of other immune cells, which leads to nonspecific inflammation.

How then should stem cells be delivered to the injured heart? Three strategies have been investigated. Transthoracic myocardial injection showed favourable outcomes in a clinical trial, ${ }^{4}$ but more recent research has focused on developing less invasive approaches such as catheter-based endomyocardial injection and intracoronary infusion. We conducted a randomized controlled clinical trial ${ }^{5}$ to evaluate the efficacy and safety of stem cell therapy in patients with myocardial infarction who underwent percutaneous coronary stenting of the infarctrelated artery. There were 3 patient groups: the first received intracoronary infusion of peripheral blood stem cells, the second received G-CSF to induce mobilization of peripheral blood stem cells (theoretically, to increase their delivery to the heart), and the third group served as a control. Interim results indicated improved cardiac function and exercise capacity in the group who received intracoronary therapy compared with the other 2 groups.

We have also observed improvement of myocardial perfusion with stem cell therapy: 
Stem cells not only differentiate into contracting cardiac myocytes but also secrete cytokines such as vascular endothelial growth factor and insulin-like growth factor that promote angiogenesis and activate resident cardiac stem cells. Indeed, in our study, improvements in coronary perfusion were relatively greater than improvements in contractility, especially in patients who received only G-CSF. The source of stem cells is likely important in this regard, given that peripheral blood stem cells contain a greater proportion of angiogenic cells than myogenic cells in vitro. We speculated that angiogenesis more than myocardial regeneration contributed to improvement in cardiac function after cell therapy, although the clinical benefit did not vary according to cell type. The benefits from other cells, such as embryonic stem cells or purified cardiac stem cells, might depend on another mechanism.

\section{Hurdles to overcome}

Although general enthusiasm for stem cell therapy for cardiac disease is fuelling intense research, we are also starting to appreciate the potential adverse effects of this novel treatment. For example, in patients who received intracoronary treatment, we observed higher rates of restenosis in the stented culprit lesions in the coronary artery. Interestingly, the degree of neointimal growth was proportional to that of improvement in cardiac function. Although restenosis was successfully managed with the additional deployment of drug-eluting stents, this served as our first warning. We have preliminary evidence from our current trial, MAGIC CellIII, that the timing of stem cell mobilization and the use of drug-eluting stents may solve the problem of restenosis caused by neointimal growth. However, other potential adverse effects have been reported in animal studies, such as microinfarction after intracoronary infusion $^{6}$ and uncontrolled differentiation of stem cells causing formation of calcification within myocardial tissue. ${ }^{?}$

Although animal studies demonstrated remarkable improvements in cardiac function with stem cell therapy, human studies have had more modest results. The increase in left ventricular ejection fraction was, although statistically significant, modest (about 5\%); this is consistent with findings from other published studies. Moreover, the long-term benefits of stem cell therapy have not been evaluated.

To proceed with and improve stem cell therapy, we need a better understanding of stem cell biology. Areas of investigation include the optimal type of cell and the kind of modification necessary, whether a heterogeneous cell population is preferable to a purified one, or differentiated cells to undifferentiated cells, the best mobilization protocol and ways to overcome the barriers to the effective homing of stem cells to the harsh environment of infarcted tissue.

\section{A promising new modality}

Currently, 2 biologic therapies for cardiovascular diseases cell and gene therapy - are under investigation in earlyphase clinical trials. Gene therapy, in general, has had limited success to date because of the short clinical effects observed and problems around the safety of the vector encoding new genetic material (typically viruses). However, gene therapy may hold the key to the future of stem cell therapy: by permitting controlled augmentation, proliferation and differentiation of stem cells through genetic manipulation, the current problems associated with stem cell therapy may be solved. Indeed, the potential benefits of stem cell therapy may not be fully realized until the advances of these seemingly divergent fields are united and give rise to an entirely new therapeutic modality.

\section{Hyun-Jae Kang \\ Hyo-Soo Kim}

Young-Bae Park

Department of Internal Medicine

Seoul National University Hospital

Seoul, South Korea

\section{References}

1. Orlic D, Kajstura J, Chimenti S, Limana F, Jakoniuk I, Quaini F, et al. Mobilized bone marrow cells repair the infarcted heart, improving function and survival. Proc Natl Acad Sci US A 2001;98(18):10344-9.

2. Orlic D, Kajstura J, Chimenti S, Jakoniuk I, Anderson SM, Li B, et al. Bone marrow cells regenerate infarcted myocardium. Nature 2001;410:701-5.

3. Menasche P, Hagege AA, Vilquin JT, Desnos M, Abergel E, Pouzet B, et al. Autologous skeletal myoblast transplantation for severe postinfarction left ventricular dysfunction. $7 \mathrm{Am}$ Coll Cardiol 2003;41:1078-83.

4. Galinanes M, Loubani M, Davies J, Chin D, Pasi J, Bell PR. Autotransplantation of unmanipulated bone marrow into scarred myocardium is safe and enhances cardiac function in humans. Cell Transplant 2004;13:7-13.

5. Kang HJ, Kim HS, Zhang SY, Park KW, Cho HJ, Koo BK, et al. Effects of intracoronary infusion of peripheral blood stem cells mobilised with granulocyte-colony stimulating factor on left ventricular systolic function and restenosis after coronary stenting in patients with myocardial infarction. Lancet 2004:363:751-6.

6. Vulliet PR, Greeley M, Halloran SM, MacDonald KA, Kittleson MD. Intracoronary arterial injection of mesenchymal stromal cells and microinfarction in dogs. Lancet 2004;363:783-4.

7. Yoon YS, Park JS, Tkebuchava T, Luedeman C, Losordo DW. Unexpected severe calcification after transplantation of bone marrow cells in acute myocardial infarction. Circulation 2004;109:3154-7. 